\title{
Numerical Analysis of Supercontinuum Generated Microstructured Optical Fiber with all Normal Chromatic Dispersion
}

\author{
Feroza Begum and Pg Emeroylariffion Abas \\ Faculty of Integrated Technologies, Universiti Brunei Darussalam, \\ Gadong BE 1410, Brunei Darussalam.
}

\begin{abstract}
A micro-structured photonic crystal fiber (M-PCF) with all normal chromatic dispersion has been proposed for supercontinuum spectrum generation which is applicable in optical transmission and optical coherence tomography applications. Calculations of its different properties using finite difference method have shown that the proposed M-PCF has a high nonlinear coefficients at $1.06 \mu \mathrm{m}, 1.30 \mu \mathrm{m}$ and $1.55 \mu \mathrm{m}$ wavelength with flattened chromatic dispersion, low confinement losses and broad supercontinuum spectrum. Moreover, it has been shown that the proposed design obtain high power and short fiber length at $1.06 \mu \mathrm{m}, 1.30$ and $1.55 \mu \mathrm{m}$ center wavelengths by propagating $\operatorname{sech}^{2}$ picosecond optical pulses with 1.0 ps pulse width at a full width at half maximum.
\end{abstract}

Key words: Microstructured fiber, chromatic dispersion, supercontinuum spectrum, longitudinal resolution

\section{INTRODUCTION}

Micro-structured fiber, also called photonic crystal fiber (PCF), has attracted considerable attention from the optical community due to its remarkable dispersion and leakage properties [1,2]. PCF has a large refractive index difference between its core and its effective cladding; and as a result, most of the energy of the fundamental mode is distributed within the core region. With light concentrated on a smaller area at a higher intensity, PCF can dramatically lower the thresholds of nonlinear optical effects.

Liao et al. [3] presented two highly nonlinear PCFs with nonlinear coefficients of $22.83 \mathrm{~W}^{-1} \mathrm{~km}^{-1}$ and $29.65 \mathrm{~W}^{-1} \mathrm{~km}^{-1}$ at the $1.55 \mu \mathrm{m}$ wavelength. The designed structures contain four different air hole diameters with hybrid claddings air holes arrangement. Doped PCF structure with nonlinear coefficients of $31.5 \mathrm{~W}^{-1} \mathrm{~km}^{-1}$ and $36.5 \mathrm{~W}^{-1} \mathrm{~km}^{-1}$ at the $1.55 \mu \mathrm{m}$ wavelength, were presented by $\mathrm{Xu}$ et al. [4] and Matloub et al. [5], respectively. A modified hexagonal index guiding photonic crystal fiber with an even higher nonlinear coefficient of $37.1 \mathrm{~W}^{-1} \mathrm{~km}^{-1}$ at the $1.55 \mu \mathrm{m}$ wavelength was presented by Hao et al. [2]. Despite the reasonably high nonlinear coefficients, their designs are difficult to fabricate; either due to the multiple air hole diameters [3], doped nature of the fiber $[4,5]$ or the presence of multiple core in the designs [2]. Additionally, nonlinear coefficients of these fibers shall be shown to be lower than that of our proposed design. Other non-hexagonal arrangements of air holes, such as square lattice structure [6], have also been proposed in the literature.

High nonlinear coefficient is also crucial for the generation of supercontinuum (SC) spectrum. Till now, super-luminescent diodes (SLDs) [7, 8], femtosecond pulse laser sources [9-12] and picosecond pulse laser source [13] have been investigated as broadband light sources. Generation of SC spectrum with $80 \mathrm{~nm}$ bandwidth and $2 \mathrm{~mW}$ to $15 \mathrm{~mW}$ output power centered at approximately $1.2 \mu \mathrm{m}$ wavelength have been demonstrated by Shibita et al. [7]. Bayleyegn et al. [8] produced an SC spectrum with $200 \mathrm{~nm}$ full width at half maxima and $10 \mathrm{~mW}$ source output power centered at $1.3 \mu \mathrm{m}$ wavelength. These output powers are, however, insufficient for the identification of individual cells [7]. Furthermore, multiplexed SLD light sources were used; with complex designs but low output power [8]. Zaytsev et al. [9] reported an SC spectrum pumped with $200 \mathrm{fs}$ Yb-doped fiber laser at

Corresponding Author: Feroza Begum, Universiti BruneiDarussalam, Gadong BE 1410, Brunei Darussalam, Ph: + 673-8653910 
central wavelength of $1.07 \mu \mathrm{m}$, to obtain $11 \mathrm{~nm}$ spectrum FWHM and $800 \mathrm{~mW}$ output power. Karim et al. [10] and Ahmad et al. [11] demonstrated the use of $1 \mathrm{~kW}$ and $100 \mathrm{~W}$ input peak power to generate SC spectrum at $1.55 \mu \mathrm{m}$ centre wavelength, with chalcogenide glass core. In reference [12], input peak power of $1.38 \mathrm{~kW}$ has been used to generate SC spectra at centre wavelength of $1.3 \mu \mathrm{m}$ and $1.65 \mu \mathrm{m}$. However, femtosecond pulse light sources are generally more expensive as compared to picosecond pulse light sources, with picosecond pulse light sources having better properties as compared to both femtosecond pulse light source and SLDs. A highly nonlinear photonic crystal fiber in OCT as a picosecond pulse laser source, is presented in reference [13]. Consequently, this paper proposes an M-PCF as a picosecond pulse laser source, due to the benefits associated with picosecond pulse laser source.

In this paper, a seven-ring M-PCF structure is proposed for supercontinuum generation with all normal chromatic dispersion. Numerical simulation results have shown that the proposed M-PCF has a high nonlinear coefficients of more than $104 \mathrm{~W}^{-1} \mathrm{~km}^{-1}, 72$ $\mathrm{W}^{-1} \mathrm{~km}^{-1}$ and $52 \mathrm{~W}^{-1} \mathrm{~km}^{-1}$ at wavelengths $1.06 \mu \mathrm{m}$, $1.30 \mu \mathrm{m}$ and $1.55 \mu \mathrm{m}$ respectively, with a very low confinement loss of less than $10^{-2} \mathrm{~dB} / \mathrm{km}$ in the 1.05 to 1.65 wavelength ranges. Moreover, the proposed MPCF exhibits an ultra-flattened chromatic dispersion of $0.0 \sim-4.5 \mathrm{ps} /(\mathrm{nm} . \mathrm{km})$ and broad supercontinuum spectrum in the targeted wavelength range, making it suitable for optical transmissions and SC generation applications. The proposed M-PCF also exhibits high power and short fiber length at center wavelength 1.06 $\mu \mathrm{m}, 1.30 \mu \mathrm{m}$ and $1.55 \mu \mathrm{m}$, which are superior to that in references [7-13].

\section{NUMERICAL SIMULATION METHODS}

Full vector finite difference method with anisotropic perfectly matched layer was used to calculate different properties of the proposed M-PCF. Chromatic dispersion $D$, chromatic dispersion slope $D_{\mathrm{s}}$, confinement loss $L_{\mathrm{c}}$, effective area $A_{\text {eff, }}$ and nonlinear coefficient $\gamma$, were some of the important parameters considered and calculated using equations (1)-(5) $[6,13$, 14]. Material dispersion, given by Sellmeier equation, has been directly included in the calculation and as such, chromatic dispersion corresponds to total dispersion of the M-PCF $[6,13,14]$.

$$
\begin{aligned}
& \mathrm{D}(\lambda)=-\frac{\lambda}{\mathrm{c}} \frac{\partial^{2} \operatorname{Re}\left[\mathrm{n}_{\text {eff }}\right]}{\mathrm{d} \lambda^{2}} \\
& \mathrm{D}_{\mathrm{S}}(\lambda)=\frac{\partial \mathrm{D}(\lambda)}{\partial \lambda} \\
& \mathrm{L}_{\mathrm{C}}=8.686 \mathrm{k}_{0} \operatorname{Im}\left(\mathrm{n}_{\mathrm{eff}}\right) \\
& \mathrm{A}_{\text {eff }}=\frac{2 \pi\left(\int_{0}^{\infty}\left|\mathrm{E}_{\mathrm{a}}(\mathrm{r})^{2}\right| \mathrm{rdr}\right)^{2}}{\int_{0}^{\infty}\left|\mathrm{E}_{\mathrm{a}}(\mathrm{r})\right|^{4} \mathrm{rdr}} \\
& \gamma=\frac{\mathrm{n}_{2} \omega}{c \mathrm{~A}_{\text {eff }}}=\frac{2 \pi \mathrm{n}_{2}}{\lambda A_{\text {eff }}}
\end{aligned}
$$

where, $\mathrm{c}$ is the velocity of light in a vacuum, $\operatorname{Re}\left(n_{\text {eff }}\right)$ and $\operatorname{Im}\left(n_{\text {eff }}\right)$ are the real and imaginary parts of complex effective index $n_{\text {eff }} \lambda$ is the operating wavelength, $E$ is the electric field, $n_{2}$ is the nonlinear refractive index coefficient and $k_{0}=2 \pi / \lambda$ is the free space wave number.

Nonlinear Schrödinger equation (NLSE) was used for numerical calculation of SC spectrum [13, 15].

$$
\begin{aligned}
& \frac{\partial A}{\partial z}+\frac{\alpha}{2} A+\frac{i}{2} \beta_{2} \frac{\partial^{2} A}{\partial T^{2}}-\frac{1}{6} \beta_{3} \frac{\partial^{3} A}{\partial T^{3}}= \\
& i \gamma\left[|A|^{2} A+i \frac{\lambda_{c}}{2 \pi c} \frac{\partial}{\partial T}\left(|A|^{2} A\right)-T_{R} A \frac{\partial|A|^{2}}{\partial T}\right]
\end{aligned}
$$

where $A$ is complex amplitude of the optical field, $z$ is propagation distance, $\alpha$ is absorption coefficient of the fiber, $\lambda_{\mathrm{c}}$ is the center wavelength, $T_{\mathrm{R}}$ is the slope of the Raman gain and $\beta_{\mathrm{n}}$ with $\mathrm{n}=1$ to 3 are the $n$-th order propagation constant. $T$ is defined as $T=$ $t-z / v_{\mathrm{g}}$ where $t$ is the physical time and $v_{\mathrm{g}}$ is group velocity at the center wavelength.

\section{STRUCTURE OF THE PROPOSED FIBER}

A schematic cross section of the proposed seven air hole rings M-PCF is shown in Fig. 1. In the model, air hole diameter are kept identical at $d$, with the exception of air hole diameters of the first and fifth rings, which have diameter of $d_{1}$ and $d_{2}$, respectively. Air hole diameter of the fifth ring $d_{2}$ is double the air hole diameter of the first ring $d_{1}$, i.e. $d_{2}=2 d_{1}$. Reductions in air hole diameters of the first and fifth rings, are due to difficulty in achieving 
near zero flattened chromatic dispersion using a conventional holey fiber structure. Distance between respective air holes or its pitch is kept at $\Lambda$. Base material of the proposed holey fiber is made up of pure silica.

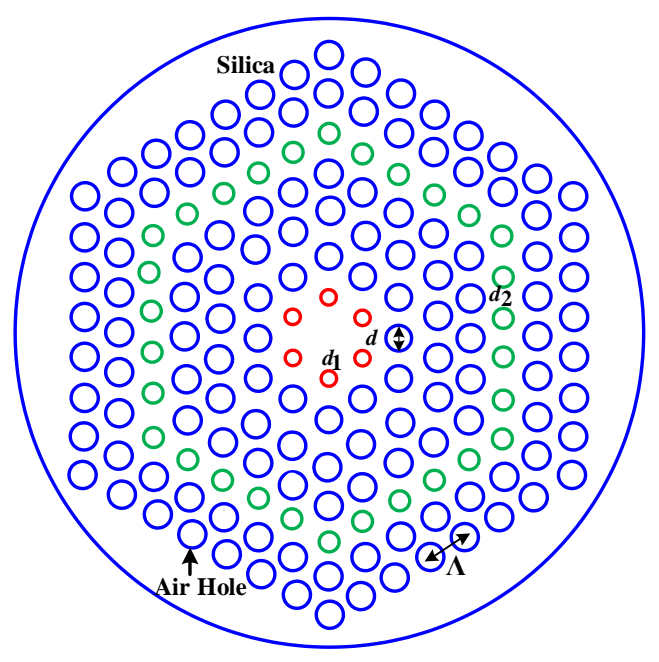

Figure 1 Proposed PCF with five rings of air hole with three different diameters $d_{1}, d_{2}, d$, and pitch $\Lambda$.

\section{RESULTS AND DISCUSSION}

For our numerical simulation, selected parameters are $d_{1}$ $=0.33 \mu \mathrm{m}, d_{2}=0.66 \mu \mathrm{m}, d=0.80 \mu \mathrm{m}$ and $\Lambda=0.87 \mu \mathrm{m}$. Fig. 2 and Fig. 3 show different properties of the proposed fiber; chromatic dispersion (Fig. 2(a)), chromatic dispersion slope (Fig. 2(b)), confinement loss (Fig. 2(c)), effective area (Fig. 3(a)) and nonlinear coefficient properties (Fig. 3(b)), for different wavelength.

It can be seen that the proposed M-PCF exhibits an ultra-flattened chromatic dispersion of $0.0 \sim-4.5$ $\mathrm{ps} /(\mathrm{nm} . \mathrm{km})$ in the observed wavelength range of 1.05 $\mu \mathrm{m}$ to $1.65 \mu \mathrm{m}$. Moreover, confinement loss is kept low below $10^{-2} \mathrm{~dB} / \mathrm{km}$ at all observed wavelength range. Effective area of the proposed M-PCF is found to be $1.78 \mu \mathrm{m}^{2}, 2.08 \mu \mathrm{m}^{2}$ and $2.42 \mu \mathrm{m}^{2}$ at wavelengths of 1.06 $\mu \mathrm{m}, 1.3 \mu \mathrm{m}$ and $1.55 \mu \mathrm{m}$ respectively. These are relatively smaller as compared to conventional fiber which commonly has an effective area of about $86 \mu \mathrm{m}^{2}$ at $1.55 \mu \mathrm{m}$ wavelength. The corresponding nonlinear coefficients are $104 \mathrm{~W}^{-1} \mathrm{~km}^{-1}, 72 \mathrm{~W}^{-1} \mathrm{~km}^{-1}$ and 52 $\mathrm{W}^{-1} \mathrm{~km}^{-1}$ at wavelength of $1.06 \mu \mathrm{m}, 1.30 \mu \mathrm{m}$ and 1.55 $\mu \mathrm{m}$, respectively; comparatively higher than nonlinear coefficients of fibers reported in references [2-6].

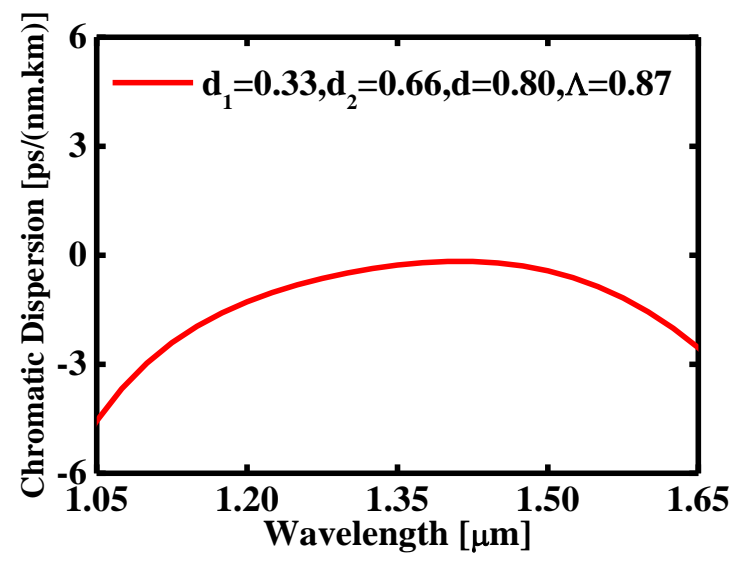

(a)

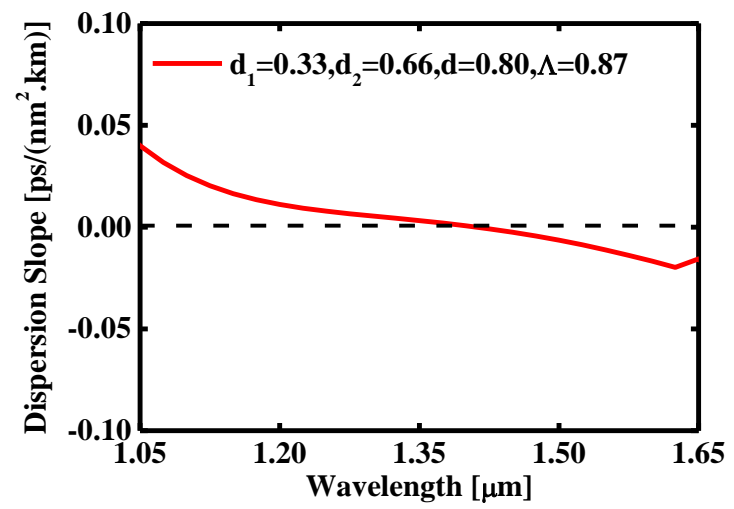

(b)

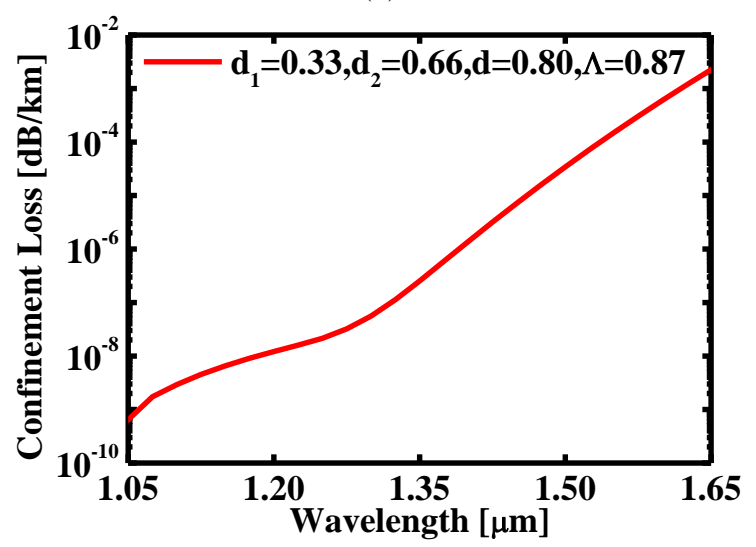

(c)

Figure 2: (a) chromatic dispersion, (b) chromatic dispersion slop and (c) confinement loss, of the proposed supercontinuum generated $\mathrm{HN}-\mathrm{PCF}$, for $\Lambda=0.87 \mu \mathrm{m}, d_{l}=$ $0.33 \mu \mathrm{m}, d_{2}=0.66 \mu \mathrm{m}$ and $d=0.80 \mu \mathrm{m}$, at different wavelength. 


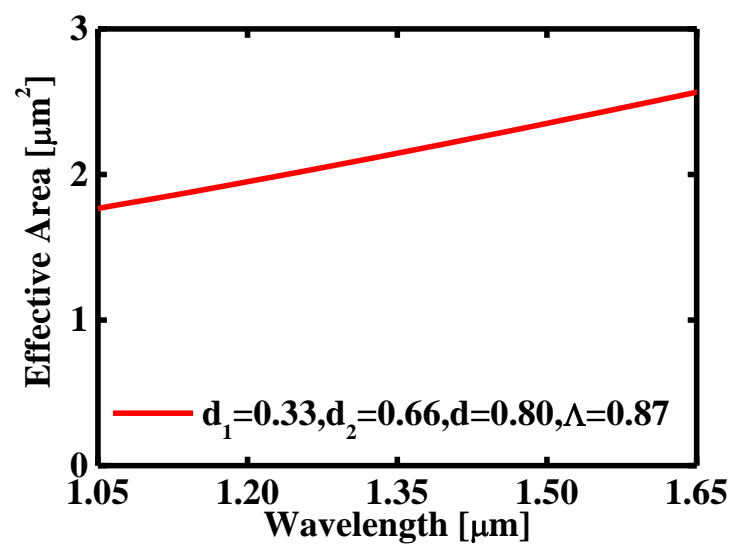

(a)

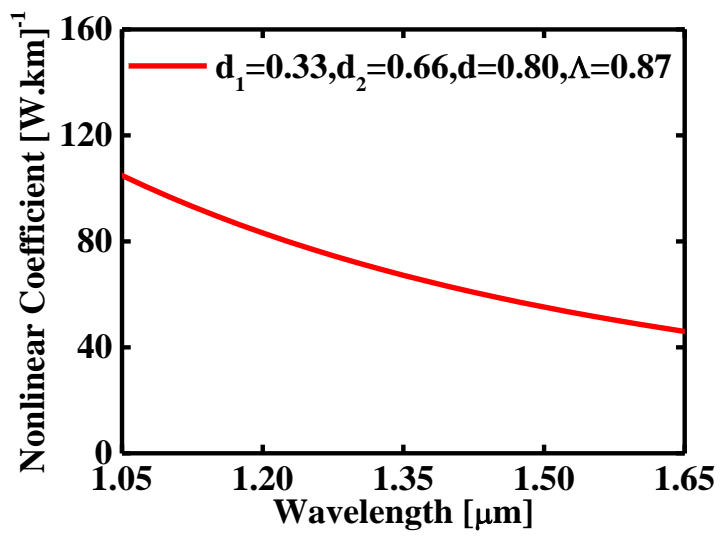

(b)

Figure 3: (a) effective area, and (b) nonlinear coefficient of the proposed supercontinuum generated $\mathrm{HN}-\mathrm{PCF}$, for $\Lambda$ $=0.87 \mu \mathrm{m}, d_{l}=0.33 \mu \mathrm{m}, d_{2}=0.66 \mu \mathrm{m}$ and $d=0.80 \mu \mathrm{m}$, at different wavelength.

NLSE was used for numerical calculation of SC spectrum [13, 15], which was solved by split-step Fourier method. SC spectrum in the proposed M-PCF was numerically calculated at $1.06 \mu \mathrm{m}, 1.30 \mu \mathrm{m}$ and $1.55 \mu \mathrm{m}$ center wavelengths which are shown in Fig. 4 (a), (b) and (c), respectively. Propagation of the $\operatorname{sech}^{2}$ waveform with full width at half maximum (FWHM), $T_{\mathrm{FWHM}}$ of $1.0 \mathrm{ps}$ and Raman scattering parameter $T_{\mathrm{R}}$ of $3.0 \mathrm{fs}$ through the proposed M-PCF was considered. Propagation constants $\beta_{2}$ and $\beta_{3}$, around the carrier frequency are shown in Table I at center wavelengths of $\lambda_{\mathrm{c}}=1.06 \mu \mathrm{m}, \lambda_{\mathrm{c}}=1.30 \mu \mathrm{m}$ and $\lambda_{\mathrm{c}}=1.55 \mu \mathrm{m}$. After numerical simulation, the incident pulse input power $P_{\text {in }}$ and fiber length $L_{\mathrm{F}}$ were obtained which are shown in Table I. The achieved input powers are $1.6 \mathrm{~kW}$ at 1.06 $\mu \mathrm{m}$ center wavelength, $2.4 \mathrm{~kW}$ at $1.30 \mu \mathrm{m}$ center wavelength and $3.8 \mathrm{~kW}$ at $1.55 \mu \mathrm{m}$ center wavelength; with acquired fiber length $L_{\mathrm{F}}$ of $6 \mathrm{~m}$ for all center wavelengths considered. These $P_{\text {in }}$ values are higher and $L_{\mathrm{F}}$ values are lower than those reported light sources in references [7-13].

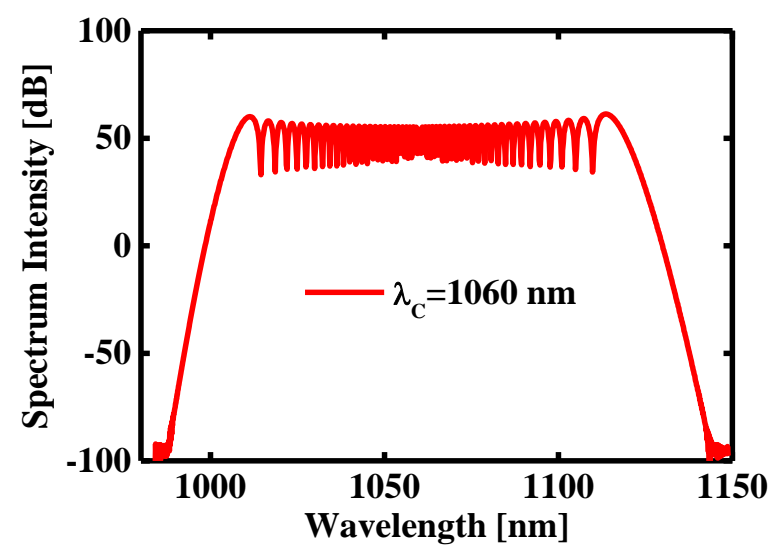

(a)

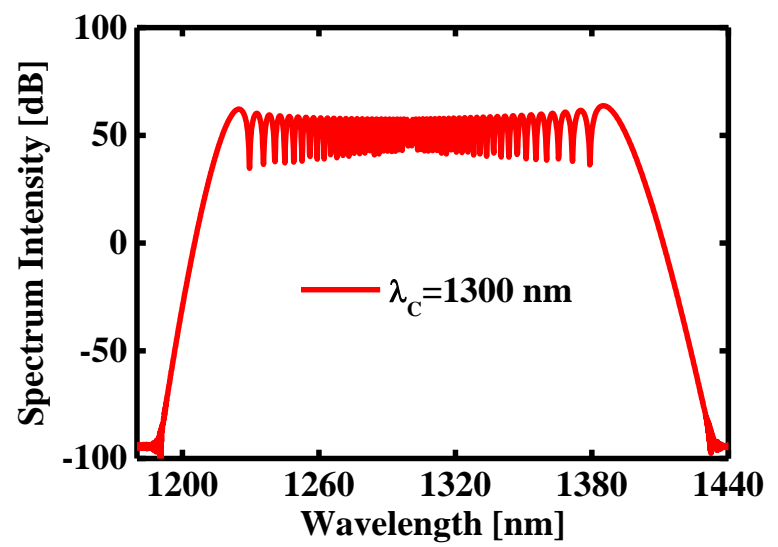

(b)

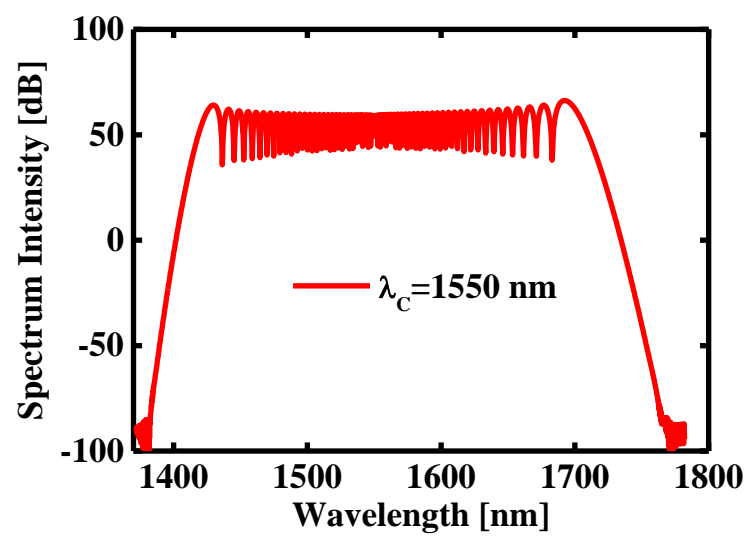

(c)

Figure 4: Spectrum intensity of the proposed supercontinuum generated $\mathrm{HN}-\mathrm{PCF}$ at center wavelengths of (a) $1.06 \mu \mathrm{m}$, (b) $1.30 \mu \mathrm{m}$ and (c) $1.55 \mu \mathrm{m}$.

Table I. Fiber parameters. 


\begin{tabular}{cccc}
\hline Parameters & $\begin{array}{c}\lambda_{c}=1.06 \\
{[\mu \mathrm{m}]}\end{array}$ & $\begin{array}{c}\lambda_{c}=1.30 \\
{[\mu \mathrm{m}]}\end{array}$ & $\begin{array}{c}\lambda_{c}=1.55 \\
{[\mu \mathrm{m}]}\end{array}$ \\
\hline$\beta_{2}\left[\mathrm{ps}^{2} / \mathrm{km}\right]$ & 2.98 & 0.318 & 2.29 \\
$\beta_{3}\left[\mathrm{ps}^{3} / \mathrm{km}\right]$ & 0.01 & 0.0045 & -0.0036 \\
$\operatorname{Pin}_{\text {in }}[\mathrm{kW}]$ & 1.6 & 2.4 & 3.8 \\
$L_{\mathrm{F}}[\mathrm{m}]$ & 6.0 & 6.0 & 6.0 \\
\hline
\end{tabular}

\section{CONCLUSION}

It has been shown that the proposed M-PCF has high nonlinear coefficient with ultra-flattened chromatic dispersion and very low confinement loss, in the wavelength range of $1.05 \mu \mathrm{m}$ to $1.65 \mu \mathrm{m}$. Moreover, the proposed M-PCF provides high longitudinal resolution and high power at $1.06 \mu \mathrm{m}, 1.30 \mu \mathrm{m}$ and $1.55 \mu \mathrm{m}$ center wavelengths, with short fiber length of $6 \mathrm{~m}$ in all center wavelengths considered. These characteristics suggest that the proposed M-PCF may be suitable for optical communications and supercontinuum generation applications, including medical imaging, tunable wavelength conversion and optical studies of photonic devices.

\section{REFERENCES}

[1] Ravi Kanth Kumar V.V., George A.K., Reeves W.H., Knight J.C., Russell P.St.J., Omenetto F.G., Taylor A.J. 2002. Extruded soft glass photonic crystal fiber for ultra-broad supercontinuum generation. Opt. Express, 10, 609-613.

[2] Hao R., Sun G. 2015. Design of photonic crystal fiber with large negative dispersion and high nonlinearity. Optik, 126, 3353-3356.

[3] Liao J., Sun J., Qin Y., Du M. 2013. Ultra flattened chromatic dispersion and highly nonlinear photonic crystal fibers with ultralow confinement loss employing hybrid cladding. Optical Fiber Technology, 19, 468-475.

[4] Xu H., Wu J., Xu K., Dai Y., Lin J. 2012. Highly nonlinear all solid photonic crystal fibers with low dispersion slope. Applied Optics, 51, 1021-1027.

[5] Matloub S., Ejlali R., Rostami A. 2015. Highly nonlinear and near zero ultra-flattened dispersion dodecagonal photonic crystal fibers. Progress In Electromagnetic Research, 60, 115-123.

[6] Begum F., Namihira Y., Razzak S.M.A., Kaijage S., Hai N. H., Zou N. 2007. Highly nonlinear dispersion-flattened square photonic crystal fiber with low confinement losses. Optical Review, 14, 120-124.
[7] Shibata H., Ozaki N., Yasuda T., Ohkouchi S., Ikeda N., Ohsato H., Watanabe E., Sugimoto Y., Furuki K., Miyaji K., Hogg R.A. 2015. Imaging of spectral-domain optical coherence tomography using a superluminescent diode based on InAs quantum dots emitting broadband spectrum with Gaussian-like shape. Japanese Journal of Applied Physics, 54, 04DG07-1-04DG07-5.

[8] Bayleyegn M.D., Makhlouf H., Crotti C., Plamann K., Dudois A. 2012. Ultrahigh resolution spectraldomain optical coherence tomography at $1.3 \mu \mathrm{m}$ using a broadband superluminescent diode light source. Optical Communications, 285, 5564-5569.

[9] Zaytsev A., Lin C.-H., You Y.-J., Chung C.-C., Wang C.-L., Pan C.-L. 2013. Supercontinuum generation by noise-like pulses transmitted through normally dispersive standard single mode fibers. Optics Express 21, 16056-16062.

[10] Karim M. R., Ahmad H., Rahman B. M. A. 2017. All-Normal Dispersion Chalcogenide PCF for Ultraflat Mid-Infrared Supercontinuum Generation. IEEE Photonics Technology Letters, 29, 17921795.

[11] Ahmad H., Karim M. R., Rahman B. M. A. 2018. Modeling of dispersion engineered chalcogenide rib waveguide for ultraflat mid-infrared supercontinuum generation in all-normal dispersion regime. Applied Physics B, 124, 47.

[12] Mohamed L.F., Lynda C., Issam H. 2018. Supercontinuum generation in silica photonic crystal fiber at $1.3 \mu \mathrm{m}$ and $1.65 \mu \mathrm{m}$ wavelengths for optical coherence tomography. Optik, 152, 106115.

[13] Begum F., Namihira Y., Kinjo T., Kaijage S. 2011. Broadband supercontinuum spectrum generated highly nonlinear photonic crystal fiber applicable to medical and optical communication systems. Japanese Journal of Applied Physics, 50, 092502092507.

[14]Zhu Z., and Brown T. 2002. Full-vectorial finitedifference analysis of microstructured optical fibers. Opt. Express, 10, 853-864.

[15] Agrawal G.P., Nonlinear Fiber Optics, Academic Press, San Diego, CA, 2nd Edition, 1995. 